\title{
Stabilized UAV Flight System Design for Structure Safety Inspection
}

\author{
Oh-hoon Cho*, Kyeong-jin Ban*, Eung-kon Kim* \\ *Department of Computer Science, Sunchon National University \\ \{worldnet, multwave, kek\}@sunchon.ac.kr \\ Corresponding Author: Eung-Kon Kim
}

\begin{abstract}
For the small air vehicle-based structure safety inspection, it is very important to collect the images without shaking and the data by manipulating the air vehicle, even though the inspector does not have professional experience. In this article, the method for the stabilized flight system design for the safety inspection of the small air vehicle-based structures.
\end{abstract}

Keywords - Quadcopter, Stabilization, UAV, Attitude controller, Optimized structure design, PWM, Autonomous Flight

\section{INTRODUCTION}

Recently, as the methods using UAV are being sought in the multipurpose surveillance and monitoring technologies such as lifesaving. collection of the weather information, the forest fire prevention, etc, the active researches are under progress in various areas [1-5]. Particularly, as a measure to minimize the cost and the loss of lives in the structure safety inspection of the high-rise building, dangerous works or work site, the technologies using UAV(Unmanned Air Vehicle) are getting the limelight. Therefore, as the importance of collecting the stabilized images without interference of shaking and the data for the structure safety inspection by manipulating UAV with radio without the operator's professional knowledge or experience is emphasized, in this study, the stabilized flight system design for the UAV-based structure stability inspection was performed.

The research objective was to design the flight stabilization system as a part of the study for the movement control module to identify the distance between the structure and the air vehicle and to maintain established altitude and distance automatically, and of the study on the automated inspection system, which performs the safety inspection for the entire structure automatically by identifying the shape of the structure.

\section{RELATEd STUdIES}

\section{A. Definition of the small unmanned air vehicle}

UAV refers to the small air vehicle, which mounts camera, communication equipments, various sensors or other equipments to manage them and is controlled remotely or controlled automatically according the pre-configured control program.

Although it is small enough that the man cannot board directly, if the operator controls directly and indirectly the air vehicle and the contents for specific tasks to be controlled are programmed, the air vehicle itself will arrive at the position and perform the tasks according to the information collected by various sensors mounted in the air vehicle.

In the current unmanned air vehicle technology areas, the most advanced area is the military weapon system area such as Hunter, Predator, Global Hawk, etc, and various areas are using low cost and minimum size of UAV such as the meteorological area to collect the weather information, the environmental area to collect the ecosystem information, the air photography for the geological information in the private industrial areas, etc.

\section{B. Type of Multi-Copter}

As one type of UAV developed by complementing the weakness of the helicopter, multi-copter is the air vehicle enabled the stable position control by rotating the props reversely without rotating fuselage, having advantages in the working area as hovering is possible and in the take-off and landing [6] as the more the number of props it has, the greater power can be obtained greater power.

Multi-copter is classified into the multi-copter and the micro-copter according to the size, and according to the number of rotors, which obtain the lift and propulsion, it is sub-classified into the dual-copter or heli-copter having 2 rotors, tri-copter having 3 rotors, quad-copter having 4 rotors, hexa-copter having 6 rotors and octo-copter having 8 rotors, as shown in Fig. 1.

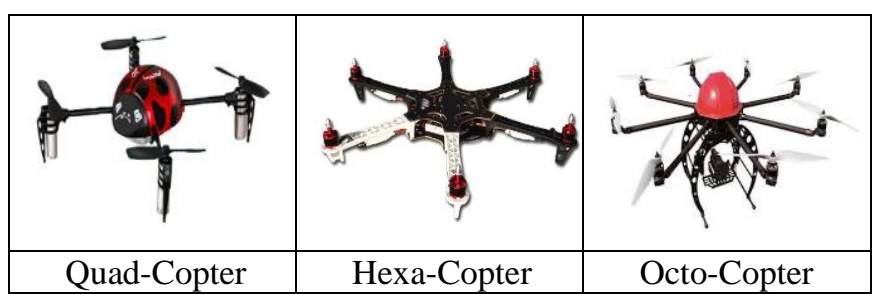

Figure 1. Classification by number of rotor 
And the unmanned flight control system to control such air vehicles is composed of 3 elements; the ground station, the communication network and the air vehicle.

\section{Blade Rotation Method}

In case of normal air vehicles, although to hover in the space, it exposes the weakness of circling large space, the blade, which is served as propeller enabled the helicopter to take off and land, can hover and perform stable flight and diverse tasks within the limited space.

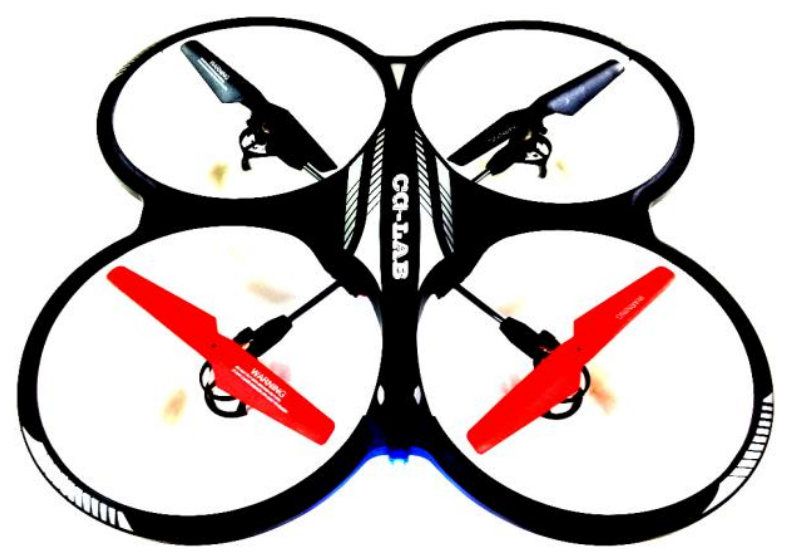

Figure 2. Quad-copter

Fig. 2 is showing the quad-copter, a kind of multi-copter classified as UAV, and the Fig. 3 shows that all the 4 blades are formed the rectangular shape, and in case of 4 blades, the blades on the diagonal line facing each other rotate to same direction, and the adjacent blades are configured to rotate to reverse direction.

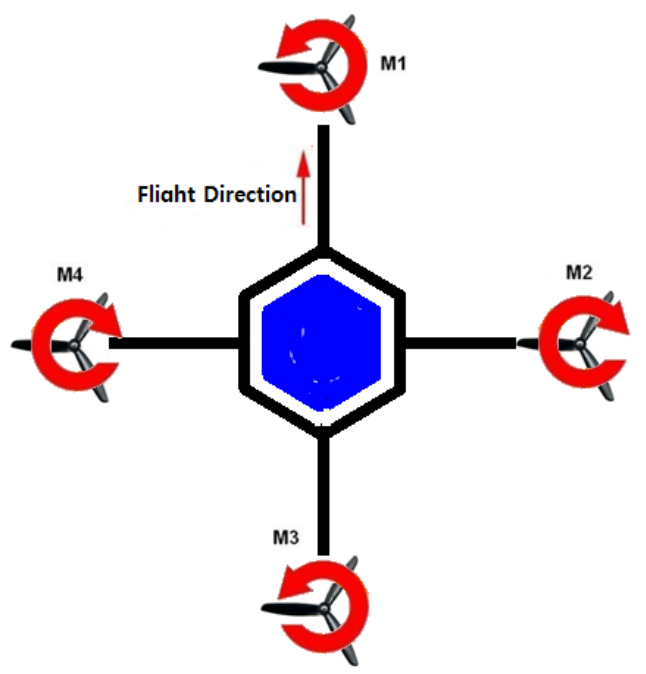

Figure 3. Blade Rotation Method

In this time, the ascending and descending of the air vehicle can be induced by changing all the blade rotation speeds according to the blade rotation method shown in Fig. 3, and by changing the front and back blade speeds (supposing the frontal direction as $\mathrm{X}$-axis), pitch moment is generated and the roll moment is generated by changing the left and right blade speeds. And if the speed is changed by controlling the left and right blades and the front and back blades in pair, yaw moment is generated. Blade motor control method is as follows.

$$
\begin{aligned}
& \text { \#elif defined(QUAD_COPTER) } \\
& \text { MotorOut } 1 \text { - = RxlnYaw; } \\
& \text { MotorOut2 + = RxlnYaw; } \\
& \text { MotorOut3 + = RxlnYaw; } \\
& \text { MotorOut4 - = RxlnYaw; }
\end{aligned}
$$

That is, since the quad-copter like UAV has very simple principle of motion, and the gyro sensor for position control, the terrestial magnetism sensor for operator-oriented control and the barometric pressure sensor to hold the altitude are mounted basically, it can be controlled skillfully without having professional knowledge on the position control of the air vehicle [7].


Figure 4. Movement Direction by Blade Rotation Method

Particularly, the recent expensive UVAs increase the operator's convenience much higher by mounting diverse sensors, perfect GPS position hold, stable flight function, excellent gimbal control function, etc.[8]. The fail safe function using GPS holding technology is the technology to prevent the risk of losing the air vehicle in the situation that the basic transmission signal between the ground station and the air vehicle is cut off, and makes hovering the air vehicle (auto pilot) until the transmission signal is reestablished or makes returning to where it took off safely (Go Home) and landing safely (Auto Landing)

\section{Contents AND Method of STUdy}

\section{A. Contents of Study}

This study was the first stage of the research to design the flight stabilization system, which controls the movement of the air vehicle accurately and easily by providing the inspector with the stabilized flight control function for the structure safety inspection, and later, as a specific research and development contents, the control method, which enables filming the images of the structure and the thermal images by moving the air vehicle up and down and from left to right 
identifying the distance between the structure and the air vehicle automatically and maintaining the programmed distance through the ultrasonic sensor, and the method to control the movement of the air vehicle to the designated altitude and position automatically for the structure safety inspection was sought. And the automated inspection system, which identifies the shape of structure and inspects the safety of the entire structure automatically, was designed, and the performance of the flight stabilization system was analyzed through the site application to the power line tower, plant smoke stack and the wind power generation facilities.

\section{B. Method of Study}

The flight stabilization system was designed by collecting the recent trends and technologies of identifying the location and the shape of the structure to be inspected through the ultrasonic sensor and GPS sensor, and by analyzing the related articles. In addition, for the air vehicle stabilization system, the research was performed by consulting with the companies specialized in UAV and the experts related to the air vehicle, and the site application and the performance analysis were performed by consulting with the UAV related companies.

In this article, the method to improve the position control performance of the air vehicle was suggested based on the quad-rotor type air vehicle, which the vertical take-off and landing is easy, mechanical structure is simple, the size is small and advantage in the performance compared to the price.

UX, which the inspector can control the movement of the air vehicle easily, and the interface data were collected, and the embedded flight stabilization software, which maintains the established distance and the altitude automatically, was developed by investigating and analyzing the flight stabilization technology status and related research areas.

The errors occurred after brief test and measurement were complemented by studying the technologies to fix the position and to prevent the collision, and the performance was analyzed through the site application to the power line tower, plant smoke stack and the wind power generation facilities.

\section{Flight Stabilization Study}

\section{A. ULTRASONIC SENSOR}

The ultrasonic sensor is used in the distance measurement using its characteristics of emitting the short high frequency pulse for certain time and being transmitted at the speed of sound in the atmosphere. The principle of calculating the distance with the objects is to calculate the distance based on the time difference when the signal is emitted from the sensor and when the eco signal is returned by running against the object, and is currently recognized as a new standard technology for automation.

The ultrasonic sensor of Microsonic is appropriate for measuring the target distance from $20 \mathrm{~mm}$ to $10 \mathrm{~m}$, and can explore in the atmosphere having full of dust or ink spray. In addition, it has characteristics of not damaging the function although the sputtering is made on the surface of the sensor, and today, the sensor having $30 \mathrm{~mm}$ of blind zone and the sensor having very narrow beam detect even the very detailed process reliably

\section{B. Autonomous Flight Position using Ultrasonic Sensor}

The air vehicle was controlled to enable the stable flight by identifying autonomous position of the small air vehicle using such characteristics of the ultrasonic sensor, and during the take-off and landing, the air vehicle was controlled in order to take off and land in stable position by detecting $3 \mathrm{~cm}-3 \mathrm{~m}$ using the $40 \mathrm{kHz}$ of ultrasonic waves.

To use the ultrasonic sensor, NaviBoard is needed. NaviBoard is the expansion board equipped with the 3 -axis gyro sensor, terrestrial magnetism sensor, acceleration sensor, barometric pressure sensor, etc. and is served to estimate the current tilt angle using appropriate position algorithm (DCM, Kalman Filter, etc).

It is observed that by making the ultrasonic wave emission angle to $0^{\circ}$, the tilt angle can be controlled according to the tilt angle to the ground.

With such experiment, the altitude was fixed to be maintained within $50 \mathrm{~cm}$ from the ground, and the inclination of the air vehicle can be controlled by the ultrasonic sensor only by removing the gyro sensor. The system was programmed in order for the 4 ultrasonic sensors to detect once each for 0.1 second to control the blade motor with 10 times/sec. 4 ultrasonic sensor were installed at the front, back, left and right below the blade to control each blade motor. In this time, the measured values under $54 \mathrm{~cm}$ and above the $55 \mathrm{~cm}$ from the ground were filtered to increase the reliability. Fig. 5 shows the system configuration diagram for the flight stabilization. The control system, which received the control signal from the ground station, received the data signal for the altitude and the tilt angel collected by the 4 ultrasonic sensors mounted in the expansion board controls the blade motor RPM(Revolutions per Minute) by comparing and analyzing the altitude and the inclination of the air vehicle according to the programmed control programm.



Figure 5. Flight Stabilization System Configuration Diagram 


\section{Ultrasonic Sensor's Tilt Measuring Test.}

Fig. 6 shows the tilt test of the air vehicle by the tilt angle of $0^{\circ}, 5^{\circ}, 10^{\circ}$ and $15^{\circ}$.



Figure 6. Air Vehicle Inclination Test

\begin{tabular}{|c|c|c|}
\hline \multicolumn{2}{|c|}{ Table 1. Air Vehicle Inclination Test } \\
\hline $\begin{array}{c}\text { Altitude of } \\
\text { Air Vehicle }\end{array}$ & $\begin{array}{r}\text { Tilt Angle to } \\
\text { the Ground }\end{array}$ & $\begin{array}{c}\text { Tilt Angle of } \\
\text { Air Vehicle }\end{array}$ \\
\hline $45 \sim 55 \mathrm{Cm}$ & $0^{\circ}$ & $0 \sim 2^{\circ}$ \\
\hline $45 \sim 55 \mathrm{Cm}$ & $5^{\circ}$ & $5 \sim 7^{\circ}$ \\
\hline $45 \sim 55 \mathrm{Cm}$ & $10^{\circ}$ & $10 \sim 12^{\circ}$ \\
\hline $45 \sim 55 \mathrm{Cm}$ & $15^{\circ}$ & $15 \sim 18^{\circ}$ \\
\hline
\end{tabular}

Table 1 shows the results of air vehicle inclination test by the tilt angle to the ground using ultrasonic sensor, and it is observed that the stable position control of the air vehicle inclination is possible from the air vehicle inclination test by the tilt angle to the ground using the ultrasonic sensor. Therefore, it is observed that the blade can control the motor speed stably. Although to measure the accurate angle, there is problem that the angles measured by the ultrasonic sensors should be matched exactly, and the reliability on the accuracy may be dropped a little bit due to the low cost observation equipments currently prepared, since the experiment was performed as closest to the maximum accuracy, the reliability for the purpose of the experiment is deemed to be enough with the approximate value.

\section{Blade Motor RPM control}

As a measure to control the flight control, the blade motor RPM was controlled after detecting the inclination and the altitude of the air vehicle using ultrasonic sensor.

Blade motor RPM was controlled according to the value obtained by converting the value measured from the ultrasonic sensor to the distance value after measuring the blade motor RPM in PWM(Pulse Width Modulation) waves. That is, as shown in Fig. 5, the stable autonomous flight was maintained by controlling the blade RPM according to the inclination of the air vehicle.

\section{Conclusion}

Although currently lots of small air vehicle such as the micro-air vehicle resembled the birds or insects including the multi-copters are developed, in real world, lots of restrictions are followed. In case of helicopter, due to difficult dynamic interpretation for automation, the experience-based control is being attempted rather than the equation-based. In this circumstances, the following vehicle was produced and the control test was performed to develop the air vehicle, which can be analyzed by flight dynamics easily and the autonomous flight is possible. And in the test results, it was controlled well enough and deemed to have potentials to be developed further.

In the results of this study, it is observed that the stabilized flight is possible using the ultrasonic sensors, and the autonomous flight, even the obstacle avoidance function, is possible by diagnosing and judging by the UAV itself when detecting unexpected obstacles during the flight to the destination for the structure inspection. The stabilized UAV flight for the structure safety inspection and the autonomous flight will be realized by performing the lots of researches continuously.

\section{ACKNOWLEDGMENT}

"This research was financially supported by the Ministry of Education (MOE) and National Research Foundation of Korea(NRF) through the Human Resource Training Project for Regional Innovation (No. 2013H1B8A2032217)."

\section{REFERENCES}

[1] A. Verma, H. Sawant and J. Tan, "Selection and Navigation of Mobile Sensor Nodes Using a "Sensor Network", Pervasive and Mobile Computing, Vol. 2 pp. $65-84,2006$

[2] Jong Hyon Hwang, Sungpil Hwang, Sung Kyung Hong, Min Goo Yoo, "Attitude Stabilization Performance Improvement of the Quadrotor Flying Robot", J.ICROS.2012, vol. 18 (6), pp.608

[3] S. Yoon, O. Soysal, M. Demirbas, and C. Qiao, "Coordinated locomotion and monitoring using "autonomous mobile sensor nodes", IEEE Transactions on Parallel and Distributed Systems, vol. 22, no. 10, pp. 1742-1756, Oct. 2011

[4] Y. Mei, C. Xian, S. Das, C. Hu, Y-H, Lu, "Sensor Replacement Using Mobile Robots", Computer Communications, Vol. 30, pp. 2615-2626.

[5] S. Abdel-Mageid and R.A. Ramadan, "Efficient deployment algorithms for mobile sensor networks", in Autonomous and Intelligent Systems (AIS), 2010 International Conference on, pp. 1-6.

[6] Jong Hyon Hwang, Sungpil Hwang, Sung Kyung Hong, Min Goo Yoo. "Stabilization performance improvement of the Quadrotor flying robot", ICROS Annual Conference 2012, vol. 41, pp. 7-9, April. 2012

[7] Sven Lange, Niko Sunderhuaf, Peter Protzel, "Autonomous Landing for a Multirotor UAV Using Vision", International Conference on 
Simulation Modeling and Programming for Autonomous Robots, 482491, 2008.

[8] Ruesch, "Dynamics Identification\& Validation, and Position Control for a Quadrotor",Semester-Thesis, Eidgenossishce Technische Hoschschule Zurich, 2010.

[9] Sung-Ho Chang, Seong-Wook Choi, Sam-Ok Koo, "Development of the Scaled Vehicle of Smart DAV," KARI vol. 6 (2), pp. 236-244, 2007

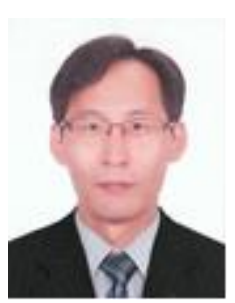

Oh-hoon Cho

Oh-hoon Cho received the B.S. degree from Korea National Open University, Korea, in 2006, His M.S. degree from department of computer science, Sunchon National University, Korea, in 2013. His currently a $\mathrm{Ph} . \mathrm{D}$. student in computer science at the Sunchon National University, Korea, His current research interests include augmented reality, image processing, computer graphics.



\section{Kyeong-Jin Ban}

Kyeong-Jin Ban received the B.S., M.S., and Ph.D degrees in computer science from Sunchon National University, Korea, in 2003, 2005, 2011, respectively. His current research interests include computer graphics, wireless sensor network, and augmented reality.

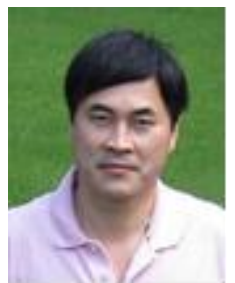

\section{Eung-kon Kim}

Eung-kon Kim received the B.S. degree from Chosun University, Gwangju,, Korea, in 1980, his M.S degree from department of electronics, Hanyang University, Seoul, Korea, in 1987, his Ph.D. degree from Chosun University, Gwangju, Korea, in 1992. His current research interests are computer vision, virtual/augmented reality, image processing, and computer graphics. Currently he is a professor in department of computer engineering, Sunchon National University, Korea. 